\title{
Genomics of tailless bacteriophages in a complex lactic acid bacteria starter culture
}

\author{
Svetlana Alexeeva ${ }^{\text {a, } 4}$, Yue Liu ${ }^{\text {a, } 4}$, Jingjie Zhu ${ }^{\text {a, } 1}$, Joanna Kaczorowska ${ }^{\text {a, }}{ }^{2}$, \\ Thijs R.H.M. Kouwen ${ }^{\text {b, }}{ }^{3}$, Tjakko Abee ${ }^{\text {a }}$, Eddy J. Smid ${ }^{\text {a, * }}$ \\ ${ }^{a}$ Food Microbiology, Wageningen University \& Research, Wageningen, the Netherlands \\ ${ }^{\mathrm{b}}$ DSM Biotechnology Center, Delft, the Netherlands
}

\section{A R T I C L E I N F O}

\section{Article history:}

Received 1 May 2020

Received in revised form

12 October 2020

Accepted 13 October 2020

Available online 21 October 2020

\begin{abstract}
A B S T R A C T
Our previous study on a model microbial community originating from an artisanal cheese fermentation starter revealed that bacteriophages not only co-exist with bacteria but also are highly abundant. Here we describe the genomic content of phage particles released by 6 different strains in the starter culture. The identified prophages belong to three different subgroups of the Siphoviridae P335 phage group. Remarkably, most analysed prophages show disruptions in different tail encoding genes, explaining the common tailless phenotype. Furthermore, a number of potentially beneficial features for the host carried by prophages were identified. The prophages carry up to 3 different phage defence systems per genome that are potentially functional in protecting the host from foreign phage infection. We suggest that the presumably defective prophages are a result of bacteria-phage coevolution and could convey advantages to host bacteria.
\end{abstract}

(C) 2020 The Authors. Published by Elsevier Ltd. This is an open access article under the CC BY license (http://creativecommons.org/licenses/by/4.0/).

\section{Introduction}

Cells from all domains of life are susceptible to viral infections. As prokaryotes outnumber eukaryotes, their viruses (bacteriophages or, simply, phages), are estimated to be the most abundant biological entities on Earth. Total number of bacteriophages is estimated to be $10^{31}$ in the biosphere (Comeau et al., 2008). Phages are ubiquitously distributed in nature and play an important role in the ecology of their bacterial hosts. In complex microbial consortia such as those found in a marine environment, the gastrointestinal tract and in complex food fermentations, bacteriophages can alter the dynamics and diversity of microbial communities (Erkus et al., 2013; Smid et al., 2014; Spus et al., 2015; Stern \& Sorek, 2011). Additionally, bacteriophages help to drive microbial evolution

\footnotetext{
* Corresponding author. Tel: +31 0317482834.

E-mail address: eddy.smid@wur.nl (E.J. Smid).

1 Current address: Department of Pathology, New York University School of Medicine, New York, NY 10016, USA.

2 Current address: Laboratory of Experimental Virology, Department of Medical Microbiology, Amsterdam UMC, University of Amsterdam, Meibergdreef 9, Amsterdam, The Netherlands.

${ }^{3}$ Current address: FrieslandCampina Laboratory \& Quality Services (LQS), Leeuwarden, The Netherlands.

4 These authors contributed equally to this work.
}

through phage-mediated gene transfer (Canchaya, Fournous, Chibani-Chennoufi, Dillmann, \& Brüssow, 2003; Penadés, Chen, Quiles-Puchalt, Carpena, \& Novick, 2015).

Bacteriophages can also occur naturally in food. Lactic acid bacteria have been used for centuries in the production of fermented food products, with for instance Lactococcus lactis as an important player in various dairy fermentations. Bacteriophages infecting $L$. lactis strains are mostly studied because of their detrimental impact on industrial milk fermentation processes (Mahony \& van Sinderen, 2015).

All described $L$. lactis phages are members of the Caudovirales order and possess a double-stranded DNA genome and a noncontractile tail. The vast majority of lactococcal phages belong to one of the three main groups within Siphoviridae family: 936, c2, or P335 (Mahony et al., 2017). Groups 936 and c2 consist of only virulent phages, while the P335 group consists of both temperate and virulent phages (Chmielewska-Jeznach, Bardowski, \& Szczepankowska, 2018). P335 phages resemble lambdoid phages, are genetically heterogeneous and have a mosaic genome structure with functional modules exchangeable through homologous recombination (Chopin, Bolotin, Sorokin, Ehrlich, \& Chopin, 2001; Desiere, Lucchini, Canchaya, Ventura, \& Brussow, 2002; Moineau, Pandian, \& Klaenhammer, 1994). Maintenance of a temperate bacteriophage inside a bacterial chromosome in the form of a 
prophage, also referred to as lysogeny, is a common phenomenon in L. lactis strains (Brøndsted \& Hammer, 2006; Kelleher et al., 2018). Lysogenic bacterial strains, carrying inducible prophages in the chromosome, usually do not find their way into commercial fermentation practice when it concerns a defined starter composition (Garneau \& Moineau, 2011). Nevertheless, lysogenic bacteria are often found in undefined mixed starter cultures.

It was discovered that a naturally evolved complex starter culture (named Ur), featured by stable composition and robustness, is composed of mainly lysogenic strains (Alexeeva, Guerra Martínez, Spus, \& Smid, 2018). Remarkably, all released bacteriophages from the complex starter culture Ur were found essentially tailless. Up to $10^{10}$ tailless phage particles per $\mathrm{mL}$ of culture are spontaneously produced by cultures of the individual strains without the occurrence of clear signs of lysis of the lysogenic strain. In this study, we subjected phage particles released from 6 representative strains to DNA sequencing followed by annotation and attribution of functions based on in silico approaches, with the objective to understand the unusual phage phenotype and explain the evolutionary relevance of such case.

\section{Materials and methods}

\subsection{L. lactis strains and bacteriophages}

Representative strains of L. lactis TIFN1-TIFN7 were used throughout this study. These strains represent isolates from different genetic lineages originally isolated from single colonies from a complex starter culture Ur and the genome sequence has been determined (Erkus et al., 2013). L. lactis TI1c is a phage cured derivative of TIFN1 described earlier (Alexeeva et al., 2018). The strains were maintained as $15 \%$ glycerol stocks at $-80{ }^{\circ} \mathrm{C}$ and routinely grown in M17 broth (OXOID) with $0.5 \%(\mathrm{w} / \mathrm{v})$ glucose or lactose addition (OXOID). Bacteriophages used in the phage sensitivity screen are namely sk1 (Chandry, Moore, Boyce, Davidson, \& Hillier, 2003), jj50 (Josephsen \& Vogensen, 1989), p2 (Higgins, Sanozky-Dawes, \& Klaenhammer, 1988), phage TN1, TN5, TN7 (Erkus et al., 2013), and DSM Phi1-DSM Phi10 (DSM Food Specialities B.V.). Details of these phages are provided in Supplementary material Table S1.

\subsection{Phage preparation and DNA isolation}

Induction of the prophages was performed using mitomycin $\mathrm{C}$ (the stock $0.5 \mathrm{mg} \mathrm{mL}^{-1}$ solution in $0.1 \mathrm{M} \mathrm{MgSO}_{4}$ was stored at $4{ }^{\circ} \mathrm{C}$ ). Overnight cultures were grown in M17 supplemented with $0.5 \%$ glucose at $30{ }^{\circ} \mathrm{C}$. The cells were diluted in fresh medium to $\mathrm{OD}_{600}=0.2$ and incubated $1-2 \mathrm{~h}$, until middle/late exponential phase was reached $\left(\mathrm{OD}_{600}=0.4-0.5\right)$. At this point mitomycin $\mathrm{C}$ solution was added to final concentration $1 \mu \mathrm{g} \mathrm{mL} \mathrm{m}^{-1}$. The cultures were further incubated for $6 \mathrm{~h}$, as $\mathrm{OD}_{600}$ was monitored hourly. Cultures were centrifuged for $15 \mathrm{~min}$ at $6000 \times \mathrm{g}$ at $4{ }^{\circ} \mathrm{C}$ and the supernatant was filter-sterilised using $0.2 \mu \mathrm{m}$ polyethersulfone (PES) filters. The phage particles in the filtrate were precipitated using $1 \mathrm{vol} 20 \%$ polyethylene glycol 8000 and $2.5 \mathrm{~m} \mathrm{NaCl}$ to 4 vol crude bacteriophage suspension. The samples were incubated overnight at $4{ }^{\circ} \mathrm{C}$ and then centrifuged at $11,000 \times g$ for $60 \mathrm{~min}$ at $4{ }^{\circ} \mathrm{C}$. Supernatants were discarded and the pellets were left to dry on clean absorbent paper.

The pellets were directly used for DNA extraction using Promega Wizard $\AA$ Genomic DNA Purification Kit. The phage pellets were resuspended in $600 \mu \mathrm{L}$ of Nuclei Lysis Solution and transferred into clean $1.5 \mathrm{~mL}$ micro-centrifuge tubes. The suspension was incubated at $80^{\circ} \mathrm{C}$ for $5 \mathrm{~min}$ and then cooled to room temperature. Three $\mu \mathrm{L}$ of RNase solution (provided with the kit) was added to each sample. The samples were mixed by inversion and incubated at $37^{\circ} \mathrm{C}$ for about $50 \mathrm{~min}$. Proteinase $\mathrm{K}\left(20 \mathrm{mg} \mathrm{mL}^{-1}\right)$ was added to a final concentration of $200 \mathrm{mg} \mathrm{L}^{-1}$ and then samples were incubated at $50{ }^{\circ} \mathrm{C}$ for $30 \mathrm{~min}$. The samples were cooled to room temperature and $200 \mu \mathrm{L}$ of Protein Precipitation Solution was added to the tubes. The tubes were vortexed vigorously for $20 \mathrm{~s}$ and incubated on ice for $5 \mathrm{~min}$. After this step the samples were centrifuged $(17,000 \times \mathrm{g}$, $3 \mathrm{~min}$, room temperature). The supernatant was transferred in a new $1.5 \mathrm{~mL}$ micro-centrifuge tube containing $600 \mu \mathrm{L}$ of isopropanol kept at room temperature. Next the samples were mixed by inversion until strands of DNA were visible, centrifuged $(17,000 \times g$, $2 \mathrm{~min}$, room temperature) and the supernatant was poured off. Six hundred microlitres of $70 \%$ ethanol (kept at room temperature) was added to the tubes. Tubes were inverted several times to wash the DNA pellet and then centrifuged $(17,000 \times \mathrm{g}, 2 \mathrm{~min}$, room temperature). Ethanol was allowed to evaporate and the tubes were dried on clean absorbent paper. The tubes were left open for $15 \mathrm{~min}$ to air dry the pellet, then $100 \mu \mathrm{L}$ of DNA Rehydratation Solution was added and DNA was rehydrated at $65{ }^{\circ} \mathrm{C}$ for $1 \mathrm{~h}$. Alternatively, the DNA was rehydrated by incubating the solution overnight at $4{ }^{\circ} \mathrm{C}$.

The isolated and rehydrated DNA was additionally purified from low molecular mass DNA species using Amicon ${ }^{\circledR}$ Ultracel $100 \mathrm{~K}$ columns (cut-off $100 \mathrm{kDa}$ or double-stranded nucleotide cutoff $>600 \mathrm{bp}$ ). DNA solution was brought to $0.5 \mathrm{~mL}$ using $5 \mathrm{~mm}$ Tris buffer $(\mathrm{pH} 8)$ and added to the column. The samples were centrifuged at $14,000 \times \mathrm{g}$ and washed 3 times with $0.5 \mathrm{~mL}$ buffer. The quality of DNA before and after micro-column purification was examined by agarose gel electrophoresis.

\subsection{Sequencing and assembly of sequences}

Library preparation using TruSeq DNA sample kit and genome sequencing was performed by BaseClear BV (Leiden, The Netherlands). A paired-end DNA library with a mean gap length size between 230 and 360 bp was constructed and the sequencing was performed using HiSeq 2500 Illumina technology (Illumina Inc, Hayward, CA, USA) using a 50-cycle or 100-cycle (proPhi7) pairedend protocol. The sequencing yielded on average $1,350,000$ reads (135 MB, for 50-cycle) and 800,000 reads (155 MB, for 100-cycle).

Next-generation assembly was performed using SeqMan NGen de novo algorithm (v12, DNAStar, USA). In 4 of 6 cases $68-88 \%$ of all reads assembled in a single contig (see Table 1 for assembly statistics). The assemblies were also checked with host genome data.

Table 1

Phage genome assembly statistics.

\begin{tabular}{|c|c|c|c|c|c|c|c|}
\hline Prophage & $\mathrm{N}$ reads & $\begin{array}{l}\text { Number } \\
\text { of contigs }\end{array}$ & $\begin{array}{l}\text { Contig } \\
\text { length raw }\end{array}$ & $\begin{array}{l}\text { Contig } \\
\text { coverage }\end{array}$ & $\begin{array}{l}\text { Background } \\
\text { average coverage }\end{array}$ & $\begin{array}{l}\% \text { reads } \\
\text { in contig }\end{array}$ & $\begin{array}{l}\mathrm{x} \text { coverage } \\
\text { above background }\end{array}$ \\
\hline proPhi1 & $5,815,949$ & 1 & 41,886 & 7211 & 59 & 68 & 121 \\
\hline proPhi2 & $3,336,448$ & 1 & 37,935 & 3175 & 27 & 75 & 119 \\
\hline proPhi4 & $2,927,954$ & 1 & 38,129 & 3106 & 28 & 88 & 112 \\
\hline proPhi5 & $2,288,894$ & 3 & 41,186 & 2292 & 40 & 88 & 58 \\
\hline proPhi6 & $2,248,210$ & 1 & 38,549 & 2189 & 38 & 82 & 58 \\
\hline proPhi7 & $1,547,972$ & 15 & 40,045 & 1146 & 47 & 29 & 24 \\
\hline
\end{tabular}


The sequences were initially subjected to automated annotation using MyRAST (Rapid Annotation Subsystem Technology (RAST)) server (Aziz et al., 2008). All predicted protein-coding genes were screened using BLASTP and Psi-BLAST algorithms against the nonredundant protein database at NCBI and for conserved motifs using InterProScan 5 (Jones et al., 2014). Where applicable, nucleotide BLAST was performed at NCBI and gene ontology (GO) annotations on the gene products were performed by UniProt "QuickGO" (UniProt Consortium, 2019).

All contigs $>600 \mathrm{nt}$ long with high coverage or $>25,000 \mathrm{nt}$ long obtained from SeqMan NGen analyses were annotated using MyRAST server (Aziz et al., 2008) and screened for phage-related features.

The genome extremities of the prophages were determined by identifying the attachment sites, followed by conformation by PCR analysis (Alexeeva et al., 2018). The sequence assembly was checked for correctness by comparison with restriction data obtained by using restriction enzymes Sca I and Ava II. The data indicated that the assembly was correct.

\subsection{Phylogenetic analysis and classification}

Alignment of sequences was run on MAFFT server. The evolutionary history was inferred by using the Maximum Likelihood method based on the Tamura-Nei model (Tamura \& Nei, 1993). The percentage of trees in which the associated taxa clustered together is shown next to the branches. Initial tree(s) for the heuristic search were obtained automatically by applying Neighbor-Joining and BioNJ algorithms to a matrix of pairwise distances estimated using the Maximum Composite Likelihood (MCL) approach, and then selecting the topology with superior log likelihood value. The tree is drawn to scale, with branch lengths measured in the number of substitutions per site (next to the branches). The analysis involved 10 and 22 nucleotide sequences. All positions containing gaps and missing data were eliminated. Evolutionary analyses were conducted in MEGA6 (Tamura, Stecher, Peterson, Filipski, \& Kumar, 2013). The tail elements are aligned to their homologous counterparts of earlier described bacteriophages using MUSCLE (Madeira et al., 2019).

\subsection{Nucleotide and protein sequences accession numbers}

The sequence data reported in the present study were deposited in GenBank database under accession nos. MN534315-MN534320. The complete genomic sequences of the P335 group phages analysed in this study are available under the following GenBank accession numbers: phage name (accession number): P335 (DQ838728), 4268 (AF489521), bIL285 (AF323668), bIL286 (AF323669), bIL309 (AF323670), BK5-T (AF176025), phiLC3 (AF242738), r1t (U38906), TP901-1 (AF304433), Tuc2009 (AF109874), and ul36 (AF349457). Prophage sequences of L. lactis SK11 (SK11-1, SK11-2, SK11-3, SK11-4 and SK11-5) were derived from (NC_008527) (Ventura et al., 2007).

\subsection{Bacteriophage sensitivity tests}

Plaque assays were conducted to quantify the phage sensitivities of wild type strains and their cured derivatives. One hundred microlitres of phage suspensions in 3-4 dilution series were mixed with $100 \mu \mathrm{L}$ overnight culture of the target bacterium. The mixtures were incubated for $10 \mathrm{~min}$ prior to adding to tubes containing $2.8 \mathrm{~mL}$ soft agar. After $24 \mathrm{~h}$ incubation at $30^{\circ} \mathrm{C}$, plaques were counted and plaque-forming units per $\mathrm{mL}$ values were calculated.
Table 2

General characteristics of the 6 phage genomes.

\begin{tabular}{lllll}
\hline Prophage & Genome size (bp) \% GC & $\begin{array}{c}\text { ORFs total } \\
\end{array}$ & & $\begin{array}{l}\text { The frequency of } \\
\text { initiation codon usage, } \\
\text { \% AUG/GUG/UUG }\end{array}$ \\
\hline proPhi1 proPhi5 & 41,249 & 35.92 & 62 & $89 / 5 / 6$ \\
proPhi2 proPhi4 & 36,976 & 35.61 & 49 & $90 / 4 / 6$ \\
proPhi6 & 37,410 & $35.4 \quad 55$ & $89 / 7 / 4$ \\
proPhi7 & 38,158 & 35.24 & 56 & $95 / 2 / 4$ \\
\hline
\end{tabular}

\section{Results}

\subsection{Genomic organisation and annotation}

Whole phage genome sequences were obtained for phage particles released by L. lactis strains TIFN1, TIFN2, TIFN4, TIFN5, TIFN6 and TIFN7 upon mitomycin C (MitC) induction. The host strains are representative isolates from different genetic lineages and the genome sequence has been determined (Erkus et al., 2013). With whole genome sequencing, two prophages were predicted for all host strains mentioned above, but one of the predicted prophages in TIFN1, TIFN5 and TIFN6 and both prophages in TIFN7 have questionable completeness (Alexeeva et al., 2018). In this study only one phage genome per strain was assembled in a single scaffold of high coverage (above $1100 \times$ ). We cannot exclude the possibility that more than one prophage from each strain was induced, but we confirmed that only one phage from each strain was released in great abundance.

The bacteriophages released by TIFN1, TIFN2, TIFN4, TIFN5, TIFN6 and TIFN7 strains were named proPhi1, proPhi2, proPhi4, proPhi5, proPhi6 and proPhi7 respectively. The genome sequence of proPhi1 was found to be identical to that of proPhi5, and the sequence of proPhi2 was found to be identical to that of proPhi4. The sequence assembly was checked for correctness by comparison with restriction analysis data obtained by using two restriction enzymes (Sca I and Ava II). The data indicated that the assembly was correct and no additional fragments were detectable, confirming that only the described prophages were released from each host in high abundance (data not shown). All prophage genome sequences with annotations have been deposited in GenBank database under accession no. MN534315-MN534320.

General characteristics of the released phages such as the genome size, the GC content and the number of open reading frames (ORFs) are summarised in Table 2. The initiation codon AUG is present in most protein-coding genes, but GUG and UUG are also found in all phage genomes albeit with lower frequency (Table 2).

Analysis of phage genomes revealed that all released phage genomes have mosaic structure, organised in two clusters of divergently transcribed genes, typical for temperate lactococcal phages. The cluster transcribed leftwards mainly comprises genes encoding functions for integration and maintenance of lysogeny. Genes encoding proteins involved in DNA replication, transcriptional regulation, packaging, structural proteins and phage release are mostly transcribed rightwards.

\subsection{Taxonomy and comparative genomics}

To establish the degree of diversity between the newly described phages and their relatedness to other lactococcal phages, a comparative genome analysis was carried out. In total, 16 published P335 phage genomes (Kelly, Altermann, Lambie, \& Leahy, 2013; Ventura et al., 2007), along with proPhi1 and 


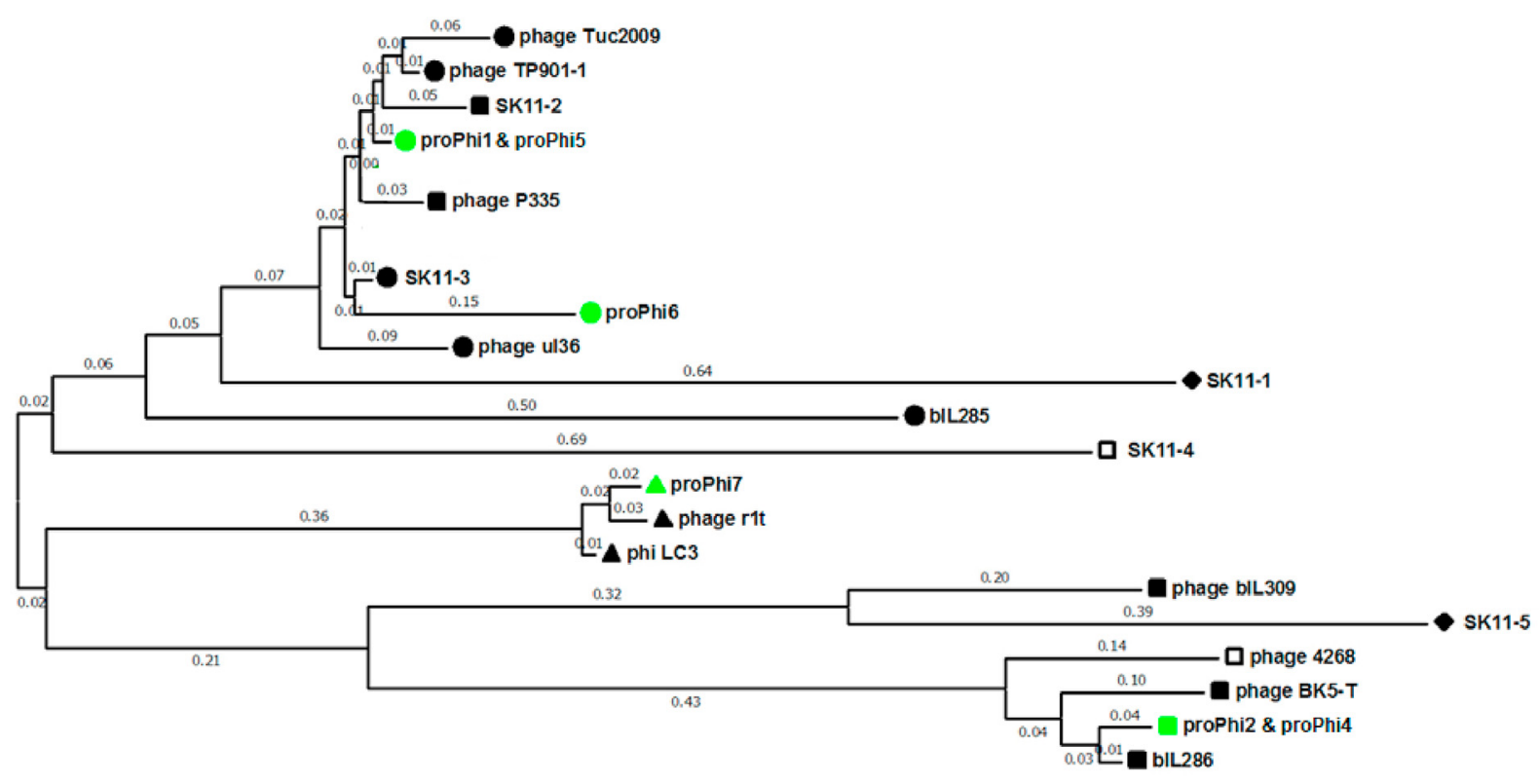

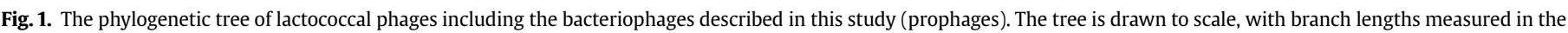

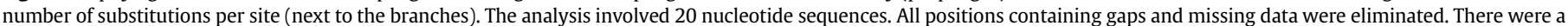

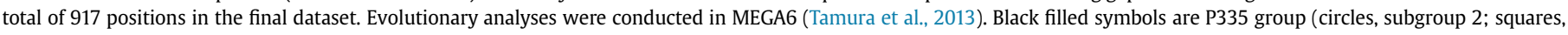

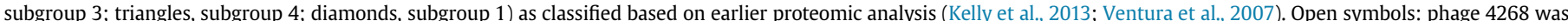

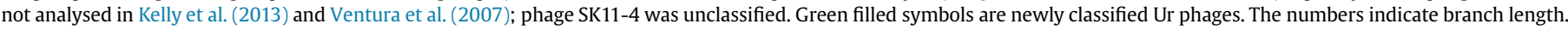
(For interpretation of the references to color in this figure legend, the reader is referred to the Web version of this article.)

proPhi5 (sequence identical), proPhi2 and proPhi4 (sequence identical), proPhi6 and proPhi7 were used to construct a phylogenetic tree (Fig. 1). The 16 phages of the lactococcal P335 group of temperate bacteriophages were selected based on earlier studies describing 4 subgroups of the P355 group lactococcal phages (Ventura et al., 2007): SK11-1 and SK11-5 belong to subgroup 1; Tuc2009, TP901-1, SK11-2, SK11-3, ul36 and bIL285 belong to subgroup 2; P335, bIL309, BK5-T and bIL286 belong to subgroup 3; r1t and LC3 belong to subgroup 4; SK11-4 and 4268 were not classified.

The phages proPhi1 and proPhi5 are most closely related to Tuc2009, TP901-1 and SK11-2. The genome sequence of proPhi6 showed most similarity to that of prophage SK11-3 and phage ul36. All of these phages fall into subgroup 2 P335 bacteriophages, all of which seem to share pac-type packaging mechanism (Labrie, Josephsen, Neve, Vogensen, \& Moineau, 2008).

The sequences of proPhi 2 and proPhi 4 clustered into subgroup 3 and proPhi7 is found among subgroup 4 bacteriophages, with bIL286 and r1t as most closely related bacteriophages respectively. The members of these subgroups share, in contrast to the subgroup 2 members, cos-type packaging mechanism (Labrie et al., 2008).

\subsection{Tail disruptions}

Thorough sequence analysis of genes encoding structural tail elements (Fig. 2) provided an explanation for the tailless phenotype of the released phage particles (Alexeeva et al., 2018; additional EM pictures in Supplementary material Fig. S1). In proPhi1/proPhi5, ORF48 and ORF51 resemble most the $\mathrm{N}$ - and $\mathrm{C}$ terminal parts of a structural tail protein in prophage SK11-2 (98 and 94\% amino acid (aa) identity, respectively) (Fig. 2A). ORF51 is $90 \%$ identical to 475 aa $C$ terminal part of tail length tape measure protein (TMP, ORF45) of phage TP901-1, a tail protein determining tail length (Pedersen, Ostergaard, Bresciani, \& Vogensen, 2000; Vegge et al., 2005).
ORF48 shares $36 \%$ identity to $\mathrm{N}$ terminal part of TMP (ORF45) of phage TP901-1. Whereas SK11-2 and TP901-1 both encode a complete protein of 874 and 937 amino acids long respectively, in proPhi1/proPhi5 the two open reading frames are separated by insertion of two mobile elements (transposase/integrase) encoded on the opposite strand (Fig. 2A). These observations suggest that ORF48 and ORF51 of proPhi1/proPhi5 encode the putative TMP that is disrupted by insertions, resulting in the tailless phenotype of the released phages.

In proPhi6 a different tail element is the target of disruption. ORF39 of proPhi6, 75 aa, is $97 \%$ identical to first 75 aa of ORF40 in Tuc2009 (102 aa) and 95\% to ORF39 in TP901-1 (103 aa). This protein seems to be highly conserved, identical proteins are also present and intact in phages ul36, P335 and in proPhi1/proPhi5 (Fig. 2B). It functions at head-tail interface and has been described earlier as putative head to tail joining protein (Brøndsted, Østergaard, Pedersen, Hammer, \& Vogensen, 2001). In proPhi6, however, the C-terminal sequence is separated from its $\mathrm{N}$-terminus by insertion of a mobile element (transposase, ORF40) encoded on the opposite strand. This fits with the observed phenotype of proPhi6: TIFN6 is the only strain that released separated tails next to the phage heads (Alexeeva et al., 2018; additional EM pictures in Supplementary material Fig. S1c) indicating that the head to tail joining function is indeed impaired.

The 3450 bp-region encompassing ORFs 44, 45, 46 of proPhi7 shares $90 \%$ nucleotide identity to ORF 40,41 and 42 of phage $\mathrm{r} 1 \mathrm{t}$ (Fig. 2C). ORF 44 and 46 in proPhi7 and ORF 40 and 42 in phage $1 \mathrm{t}$ encode the $\mathrm{N}$ and $\mathrm{C}$-terminus of a TMP. It has been suggested that r1t ORF41, identical to proPhi7 ORF45 and separating $\mathrm{N}$ and $\mathrm{C}$ terminus of the TMP, belongs to $\mathrm{HNH}$ homing endonuclease or a group I introns (McGrath et al., 2006; van Sinderen et al., 1996). However, r1t has been shown to possess a tail (Lowrie, 1974). On the other hand, phage phiLC3 encodes 843 aa long TMP, identical to r1t and proPhi7, but not interrupted by an insertion (Fig. 2C). It is 
A proPhil TMP::
Sequence Ruler
Features

SK11-2 TMP (LACR_1146)

Sequence Ruler

Features

TP901-1 TMP orf45

Sequence Ruler

Features

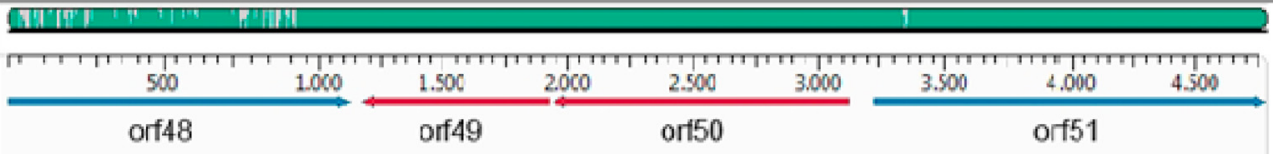

\begin{tabular}{|c|c|}
\hline 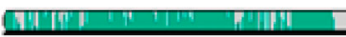 & 11 \\
\hline $\begin{array}{cccc}1+1 & \\
500 & 1000\end{array}$ & $\begin{array}{cc}1.500 & 1000\end{array}$ \\
\hline
\end{tabular}

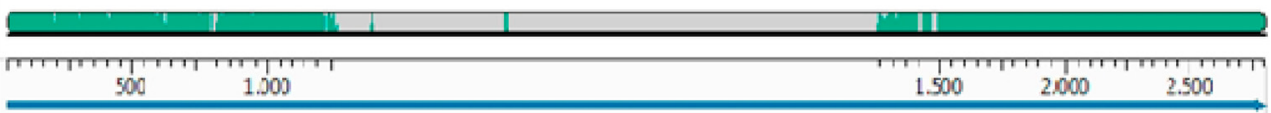

B

proPhi6 HTJ:
Features
Sequence Ruler
TuC2009 HTJ orf 40
Features
Sequence Ruler

TP901-1 THJ arf39

Features

Sequence Ruler

proPhil HTJ orf42

Features

Sequence Ruler

C

proPhi7 TMP:
Sequence Ruler
Features

philC3 TMP orf41

Sequence Ruler

Features

r1t TMP orf $40-42$

Sequence Ruler

Features
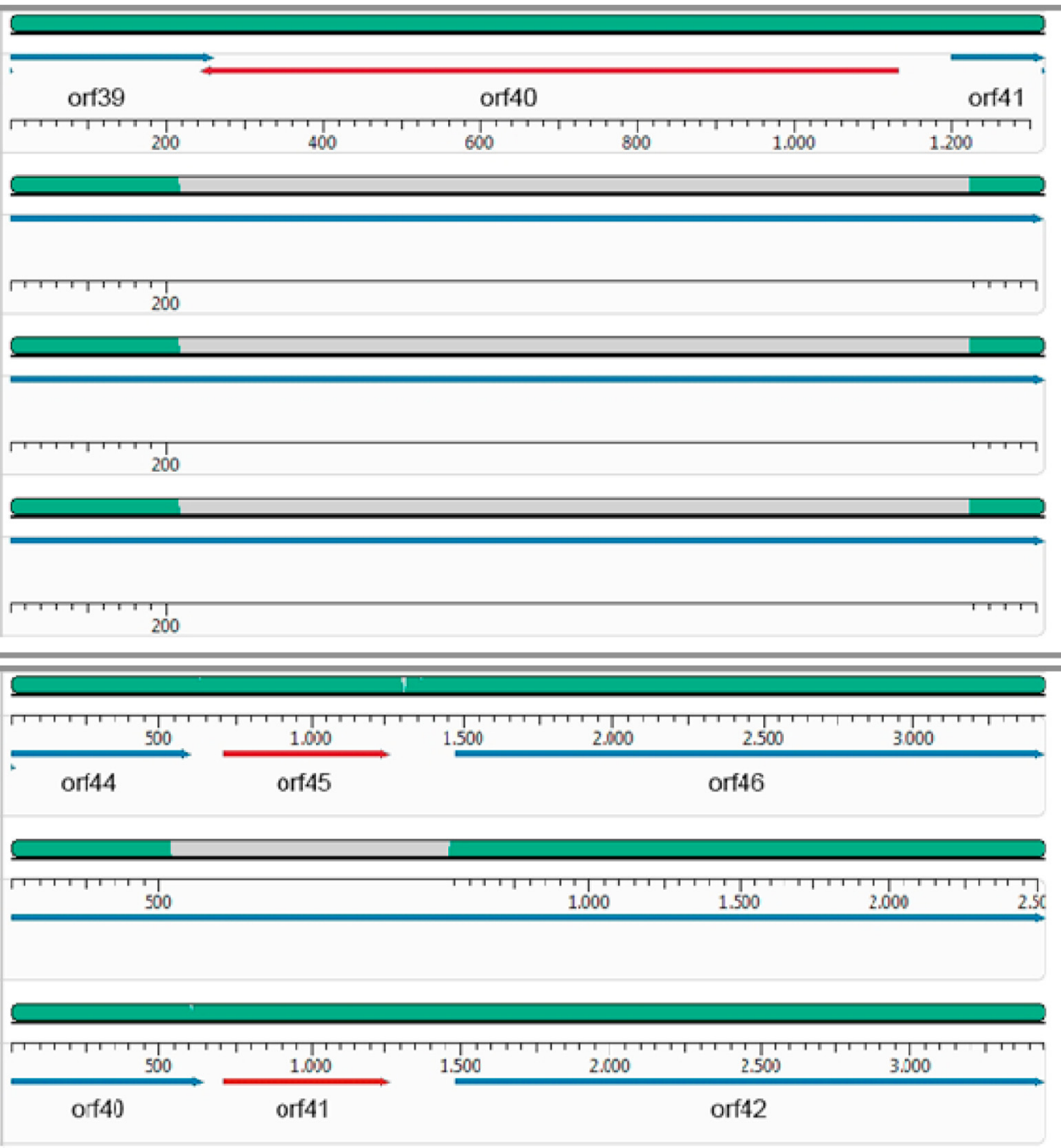

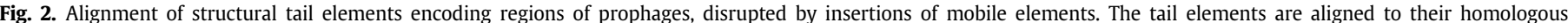

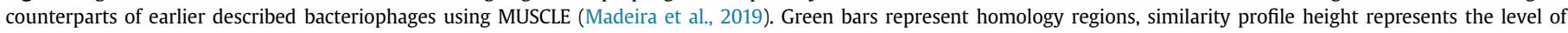

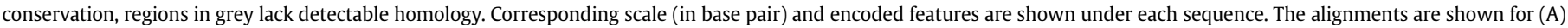

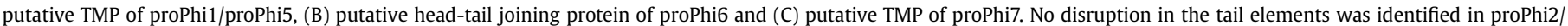

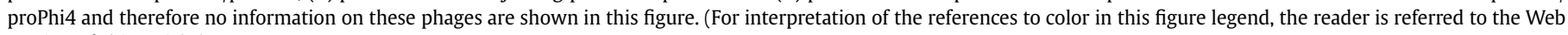
version of this article.) 
Table 3

Phage-defence protein encoding genes identified in Ur prophages.

\begin{tabular}{|c|c|c|c|}
\hline Prophage & ORF & Putative product & Defense mechanism \\
\hline \multirow[t]{3}{*}{ proPhi1/proPhi5 } & ORF2 & Sie protein & superinfection-exclusion \\
\hline & ORF61 & Abi-like protein (AbiD/AbiF) & abortive infection \\
\hline & ORF62 & N-4/N-6 DNA methylase & restriction modification \\
\hline \multirow[t]{2}{*}{ proPhi7 } & ORF55 & abortive phage resistance protein (AbiEii) & abortive infection \\
\hline & ORF56 & abortive phage resistance protein (AbiLii) & abortive infection \\
\hline
\end{tabular}
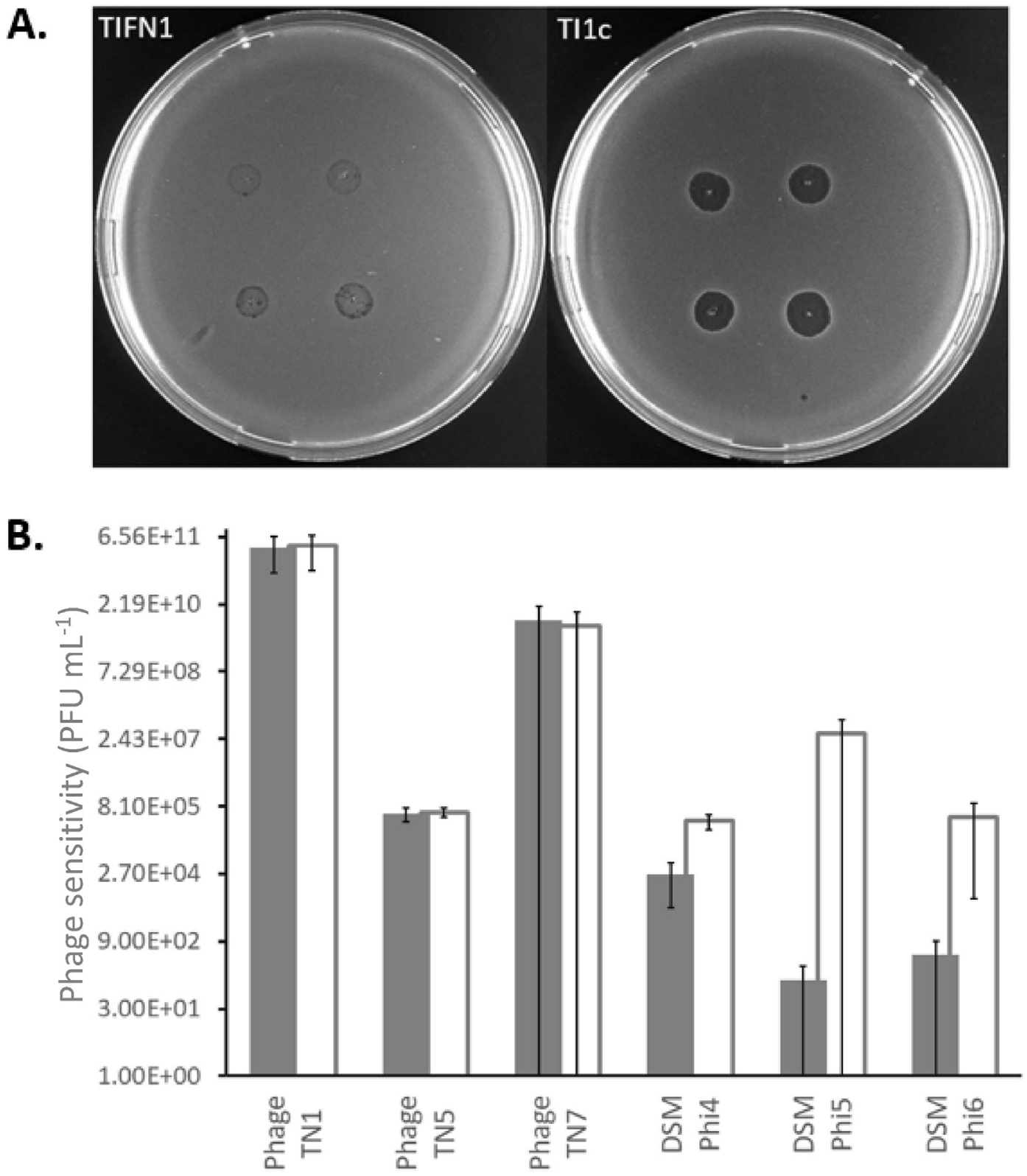

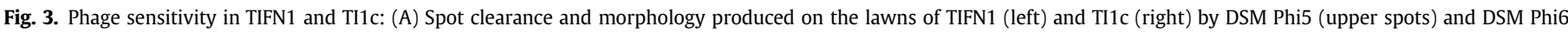

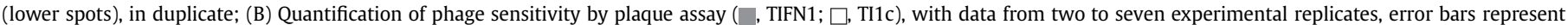
standard errors.

therefore unclear, whether or not the insertion between $\mathrm{N}$ and $\mathrm{C}$ terminus of proPhi7 TMP results in the tailless phenotype of proPhi7. In addition, no obvious disruptive elements could be identified in the tail module of proPhi2/proPhi 4 despite the observed tailless phage morphology.

\subsection{Potentially beneficial prophage encoded features}

Next to the obvious phage-related features, e.g. integration, the regulation of lytic/lysogenic conversion and structural proteins, the prophages also encode proteins with potential benefits to the host. 
Phage-defence protein encoding genes are among the most frequently observed ones (Table 3 ). ORF2 in proPhi1/proPhi5 is $98 \%$ identical to the gene encoding Sie family SieIL409 protein of Lactococcus phage 409 , and SieIL409 has been shown to mediate phage resistance by a DNA injection blocking mechanism (Mahony, McGrath, Fitzgerald, \& van Sinderen, 2008; McGrath, Fitzgerald, \& van Sinderen, 2002). The terminal ORF62 in proPhi1/proPhi5 is also assigned to have methyltransferase activity by GO annotation and is possibly part of a restriction-modification system. ORF61 of proPhi1/proPhi5 codes for an abortive infection AbiD/AbiF-like protein, showing the conserved domain of Abi_2 protein superfamily and about 50\% identity to the amino acid sequences of abortive infection bacteriophage resistance proteins in several Streptococcus phages.

The Abi system is another feature potentially involved in a phage defense mechanism: it allows phage absorption and phage DNA injection but interfere with further phage development, so that the death of infected cells occurs but no viral progeny is released (Labrie, Samson, \& Moineau, 2010). Also proPhi7 carries two genes (ORF55 \& ORF56) encoding Abi-like proteins near the attR terminus: ORF55 shows the conserved domain of AAA_21 protein superfamily (AbiEii proteins) and $30-50 \%$ identity to the amino acid sequences of abortive phage resistance proteins in several Lactobacillus phages; ORF56 shows the conserved domain of RloB protein superfamily (AbiLii proteins) and up to $40 \%$ identity to the amino acid sequences of RloB domain-containing protein in plenty members of the Firmicutes.

Furthermore, proPhi6 carries a gene for a membrane protein related to a metallopeptidase (ORF49, with $80 \%$ identity to ORF53 in proPhi1/proPhi5). The product of ORF33 in proPhi2/proPhi4 is a putative protease (ATP-dependent serine endopeptidase, $\mathrm{ClpP}$ ) and finally proPhi6 possesses prepillin peptidase (ORF13) encoding gene. Whether products of these genes could offer competitive advantages to the hosts in a microbial community by inhibiting other species remains to be elucidated.

The variety of phage resistance genes carried by prophage genomes suggests that the prophages likely contribute to host's ability to counteract superinfections. To assess this contribution we challenged lysogenic wild-type strains TIFN1 in parallel with its phage-cured derivative TI1c with 16 lactococcal phages (Supplementary material Table S1).

Six phages (phage TN1, TN5, TN7, DSM Phi4, DSM Phi5 and DSM Phi6) from the phage collection were lysis-positive for TIFN1 and TI1c as revealed by spot tests, and clear differences in sensitivity of the two strains were already visualised towards some of the phages (example Fig. 3A). Further, phage sensitivity towards the 6 lysispositive phages was quantified for the two strains (Fig. 3B). TIFN1 showed significantly $(p<0.1)$ lower phage sensitivity towards phages DSM Phi4, DSM Phi5 and DSM Phi6 compared to TI1c. Moreover, it was also noticed that the plaques were smaller and more opaque on TIFN1 than TI1C for these 3 phages (Supplementary material Fig. S2).

\section{Discussion}

In our previous study we described morphologically tailless bacteriophages, abundantly and continuously released by all analysed $L$. lactis strains originating from a complex dairy starter culture Ur without showing obvious cell lysis (Alexeeva et al., 2018). Because of their distinct morphology, behaviour and no apparent impact on host cell integrity, we hypothesized that these bacteriophages belong to a separate (novel) group of temperate lactococcal phages. However, detailed genome analysis of the released phages presented here, revealed that the bacteriophages possess a typical lactococcal P335 group Siphoviridae family genome structure, and that the phages fall under three different known subgroups of P335 phages (Ventura et al., 2007).

Temperate lactococcal phages of Siphoviridae family, belonging to the P335 group, are usually characterised by a long noncontractile tail - a structure responsible for host recognition, adsorption and the initiation of phage infection by envelope penetration and DNA ejection (McGrath et al., 2006). Despite the fact that all inducible prophages found in strains from the complex starter culture Ur so far are essentially tailless, phage sequencing revealed the presence of genes encoding most of the tail structural elements: head-tail connector, major tail protein (MTP), tail length tape-measure protein (TMP), distal tail protein, tail associated lysin, upper and lower base plate protein (Stockdale et al., 2015; Veesler \& Cambillau, 2011; Veesler et al., 2012; Vegge et al., 2005). However, detailed sequence analysis identified the presence of insertions of mobile genetic elements in the tail module in most of the phage genomes analysed in this study. Furthermore, the insertions occurred at different sites of the tail module: proPhi1, proPhi5 and proPhi7 contained (different) insertions in the TMP while proPhi6 in the head-tail joining protein. TMP determines the length of the phage tail (Katsura, 1987; Pedersen et al., 2000) and serves as a component of the precursor complex, involved in the initiation of polymerization of MTP. However, it has been shown for $\lambda$ phage that in the absence of TMP, MTP polymerisation may be initiated but the formation of tail-tube related structure is abolished (Katsura, 1976). The tailless morphology of proPhi2/proPhi4 could not be explained by this sequence analysis as no obvious disruptive elements could be identified in the tail modules. It is postulated that the absence of tails for the phage proPhi2/proPhi 4 belonging to these particular L. lactis strains is caused by modifications at transcriptional and translational level of the phage genes, or minor mutations in gene sequence.

Bacteria-phage coevolution has been regarded as an important driver of evolutionary processes and an essential player in shaping of microbial communities (Koskella \& Brockhurst, 2014). This is also reflected in the results of the phage genomics analysis performed in this study. In the Ur strains, most of the inducible prophages have mutational insertions in different tail encoding genes, resulting in tailless phage particles that are likely to be defective to infect their hosts as the tail is required for adhesion and subsequent injection of phage DNA, which is possibly a host strategy to minimize the phage impact. In fact, defective prophages are commonly observed in bacterial genomes. Out of more than 200 prophages from 83 bacterial genomes analysed in a study (Casjens, 2003), only 9 prophages were experimentally shown to be fully functional. All other prophages were found to have experienced different levels of mutational decay. Moreover, it is also acknowledged that many genes in the defective prophages remain functional and contribute to various traits of the hosts, and that the prophage functions are a result of purifying selection in the bacterial chromosome (Bobay, Touchon, \& Rocha, 2014; Kelleher et al., 2018). Prophage genes encoding core phage-related functions, e.g. tail and lysis proteins, were found to be under stronger purifying selections (Bobay et al., 2014), presumably due to the critical functions in phage spreading or host integrity carried by these genes. This finding coincides with our observation that prophages in bacterial community members of the dairy starter culture Ur, showed disruptions in the tail protein encoding genes. This could be explained, as the loss of the key structure to re-infect the same bacterial species was likely advantageous for the species to be maintained in the microbial community. In addition, the current genetic analysis also predicted prophage-encoding phage resistance systems in some of the Ur prophages, which could be linked to the phage resistance phenotype of the host; this could be also a result of purifying selection. 
Another uncommon phenotype of the studied Ur phages was the spontaneous, continuous release of phage particles, even when no stress or prophage-inducing condition was applied (Alexeeva et al., 2018). For lambda phage the spontaneous excision rates are approximately $10^{-6}$ per cell division (Bobay et al., 2014; Gottesman \& Yarmolinsky, 1968) while Muhammed et al. (2018) observed up to $10^{7} \mathrm{~mL}^{-1}$ spontaneous release of P335 phages. It is remarkable that up to $10^{10} \mathrm{~mL}^{-1}$ phage particles are released by Ur strains spontaneously (Alexeeva et al., 2018). Attempts to explain this phenotype were made by examining the repressor sequences in the prophages. In all the prophages, a repressor has been identified: orf3 in proPhi1 and proPhi5, orf2 in proPhi2 and proPhi4, orf3 in proPhi7 and orf4 in proPhi7. All identified repressors showed homology to full-length repressor genes in other phages and we did not identify obvious disrupting elements or mutations leading to premature stop codons. However, as described by Kot, Kilstrup, Vogensen, and Hammer (2016), even single point mutations in the CI repressor of phage TP901-1 were enough to affect the maintenance of the lysogenic state. It is therefore plausible that the phage-host coevolution has resulted in (minor) mutations in sequence, or modifications in the transcriptional or translational levels in the regulatory elements, that allow continuous assembly of these phage particles. Notably, the fitness of the host does not seem to be compromised by the continuous phage release, when comparing the growth performance of TIFN1 to its proPhi1-cured derivative TI1c based on OD measurement (Supplementary material Fig. S3).

It should be noted that although prophage-encoded phageresistance elements were predicted and TIFN1 showed higher phage resistance than its phage-cured derivative, this study does not intend to provide direct conclusions on the functionality of the phage-resistance elements but to provide possible explanations based on the genome data available. The phage-resistance phenotype in the prophage-harbouring strain could also be a result of self-immunity mediated by repressors, or the continuous phage producing phenotype inhibits successful infection of other incoming phages by competing and interfering with the assembly process.

All these could serve as the explanation of the phenomenon that in the bacterial community of the dairy starter Ur, presumably after a long-term selection, predominantly strains with a prophage sequence were maintained. In conclusion, the analysis of the genomic content of phage particles released by 6 different strains in the starter culture Ur provided insights for bacteria-phage coevolution, and this may also provide new leads in for future research and implications in practise, for example in defining strain selection criteria in (dairy) industry, where traits like phage resistance are desired.

\section{Authors' contributions}

SA and EJS conceived the study. SA, EJS and YL designed the experiments. SA and YL executed the experiments and carried out the data analysis and interpretation. SA, YL, TA and EJS wrote the manuscript. JZ performed phage sensitivity tests, JK participated in purification of phage DNA, TK co-designed phage sensitivity tests. All authors read and approved the final manuscript.

\section{Declaration of competing interest}

The authors declare no conflict of interest.

\section{Acknowledgements}

This work was supported by Top Institute Food and Nutrition (TIFN) in Wageningen, the Netherlands through contract FF001. In addition, YL was subsidized by the Netherlands Organisation for Scientific Research (NWO) through the Graduate Program on Food Structure, Digestion and Health. The authors are grateful to Laurens Hanemaaijer of DSM for technical support in phage sensitivity tests, acquiring and sharing of phages DSM Phi1-DSM Phi10 suspension stocks, Michiel Wels for support with sequence assemblies and Anne de Jong for support with sequence annotations. We also thank students of Wageningen University for their contribution: Venera Proneva for purification of proPhi4, and proPhi5, and proPhi6 DNA; Yixin Ge for purification of proPhi7 DNA. We thank Dr Jennifer Mahony of University College Cork, Cork, Ireland for kindly providing us bacteriophages sk1, p2 and jj50. We also gratefully acknowledge Jan van Lent [Wageningen Electron Microscopy Centre (WEMC)] for his help with electron microscopy. The datasets used and/or analysed during the current study are available from the corresponding author on reasonable request.

\section{Appendix A. Supplementary data}

Supplementary data to this article can be found online at https://doi.org/10.1016/j.idairyj.2020.104900.

\section{References}

Alexeeva, S., Guerra Martínez, J. A., Spus, M., \& Smid, E. J. (2018). Spontaneously induced prophages are abundant in a naturally evolved bacterial starter culture and deliver competitive advantage to the host. BMC Microbiology, 18, Article 120.

Aziz, R. K., Bartels, D., Best, A. A., DeJongh, M., Disz, T., Edwards, R. A., et al. (2008). The RAST server: Rapid annotations using subsystems technology. BMC Genomics, 9, Article 75

Bobay, L., Touchon, M., \& Rocha, E. P. (2014). Pervasive domestication of defective prophages by bacteria. Proceedings of the National Academy of Sciences of the United States of America, 111, 12127-12132.

Brøndsted, L., \& Hammer, K. (2006). Phages of Lactococcus lactis. In R. Calendar (Ed.), The bacteriophages (pp. 572-592). Oxford, UK: Oxford University Press.

Brøndsted, L., Østergaard, S., Pedersen, M., Hammer, K., \& Vogensen, F. K. (2001). Analysis of the complete DNA sequence of the temperate bacteriophage TP9011: Evolution, structure, and genome organization of lactococcal bacteriophages. Virology, 283, 93-109.

Canchaya, C., Fournous, G., Chibani-Chennoufi, S., Dillmann, M.-L., \& Brüssow, H. (2003). Phage as agents of lateral gene transfer. Current Opinion in Microbiology, $6,417-424$.

Casjens, S. (2003). Prophages and bacterial genomics: What have we learned so far? Molecular Microbiology, 49, 277-300.

Chandry, P. S., Moore, S. C., Boyce, J. D., Davidson, B. E., \& Hillier, A. J. (2003). Analysis of the DNA sequence, gene expression, origin of replication and modular structure of the Lactococcus lactis lytic bacteriophage sk1. Molecular Microbiology, 26, 49-64.

Chmielewska-Jeznach, M., Bardowski, J. K., \& Szczepankowska, A. K. (2018). Molecular, physiological and phylogenetic traits of Lactococcus 936-type phages from distinct dairy environments. Scientific Reports, 8, Article 12540.

Chopin, A., Bolotin, A., Sorokin, A., Ehrlich, S. D., \& Chopin, M. (2001). Analysis of six prophages in Lactococcus lactis IL1403: Different genetic structure of temperate and virulent phage populations. Nucleic Acids Research, 29, 644-651.

Comeau, A. M., Hatfull, G. F., Krisch, H. M., Lindell, D., Mann, N. H., \& Prangishvili, D. (2008). Exploring the prokaryotic virosphere. Research in Microbiology, 159, $306-313$.

Desiere, F., Lucchini, S., Canchaya, C., Ventura, M., \& Brussow, H. (2002). Comparative genomics of phages and prophages in lactic acid bacteria. Antonie van Leeuwenhoek, 82, 73-91.

Erkus, O., de Jager, V. C., Spus, M., van Alen-Boerrigter, I. J., van Rijswijck, I. M., Hazelwood, L., et al. (2013). Multifactorial diversity sustains microbial community stability. The ISME Journal, 7, 2126-2136.

Garneau, J. E., \& Moineau, S. (2011). Bacteriophages of lactic acid bacteria and their impact on milk fermentations. Microbial Cell Factories, 10, Article S20.

Gottesman, M. E., \& Yarmolinsky, M. B. (1968). Integration-negative mutants of bacteriophage lambda. Journal of Molecular Biology, 31, 487-505.

Higgins, D. L., Sanozky-Dawes, \& Klaenhammer, T. R. (1988). Restriction and modification activities from Streptococcus lactis ME2 are encoded by a selftransmissible plasmid, pTN20, that forms cointegrates during mobilization of lactose-fermenting ability. Journal of Bacteriology, 170, 3435-3442.

Jones, P., Binns, D., Chang, H.-Y., Fraser, M., Li, W., McAnulla, C., et al. (2014). InterProScan 5: Genome-scale protein function classification. Bioinformatics, 30, 1236-1240.

Josephsen, J., \& Vogensen, F. K. (1989). Identification of three different plasmidencoded restriction/modification systems in Streptococcus lactis subsp. cremoris W56. FEMS Microbiology Letters, 59, 161-166. 
Katsura, I. (1976). Isolation of $\lambda$ prophage mutants defective in structural genes: Their use for the study of bacteriophage morphogenesis. Molecular and General Genetics, 148, 31-42.

Katsura, I. (1987). Determination of bacteriophage lambda tail length by a protein ruler. Nature, 327, 73-75.

Kelleher, P., Mahony, J., Schweinlin, K., Neve, H., Franz, C. M., \& van Sinderen, D. (2018). Assessing the functionality and genetic diversity of lactococcal prophages. International Journal of Food Microbiology, 272, 29-40.

Kelly, W. J., Altermann, E., Lambie, S. C., \& Leahy, S. C. (2013). Interaction between the genomes of Lactococcus lactis and phages of the P335 species. Frontiers in Microbiology, 4, Article 257.

Koskella, B., \& Brockhurst, M. A. (2014). Bacteria-phage coevolution as a driver of ecological and evolutionary processes in microbial communities. FEMS Microbiology Reviews, 38, 916-931.

Kot, W., Kilstrup, M., Vogensen, F. K., \& Hammer, K. (2016). Clear plaque mutants of lactococcal phage TP901-1. PloS One, 11, Article e0155233.

Labrie, S. J., Josephsen, J., Neve, H., Vogensen, F. K., \& Moineau, S. (2008). Morphology, genome sequence, and structural proteome of type phage P335 from Lactococcus lactis. Applied and Environmental Microbiology, 74, 4636-4644.

Labrie, S. J., Samson, J. E., \& Moineau, S. (2010). Bacteriophage resistance mechanisms. Nature Reviews Microbiology, 8, 317-327.

Lowrie, R. (1974). Lysogenic strains of group N lactic streptococci. Applied Microbiology, 27, 210-217.

Madeira, F., Park, Y. M., Lee, J., Buso, N., Gur, T., Madhusoodanan, N., et al. (2019). The EMBL-EBI search and sequence analysis tools APIs in 2019. Nucleic Acids Research, 47, W636-W641. https://doi.org/10.1093/nar/gkz268

Mahony, J., McGrath, S., Fitzgerald, G. F., \& van Sinderen, D. (2008). Identification and characterization of lactococcal-prophage-carried superinfection exclusion genes. Applied and Environmental Microbiology, 74, 6206-6215.

Mahony, J., Oliveira, J., Collins, B., Hanemaaijer, L., Lugli, G. A., Neve, H., et al. (2017). Genetic and functional characterisation of the lactococcal P335 phage-host interactions. BMC Genomics, 18, Article 146.

Mahony, J., \& van Sinderen, D. (2015). Novel strategies to prevent or exploit phages in fermentations, insights from phage-host interactions. Current Opinion in Biotechnology, 32, 8-13.

McGrath, S., Fitzgerald, G. F., \& van Sinderen, D. (2002). Identification and characterization of phage-resistance genes in temperate lactococcal bacteriophages. Molecular Microbiology, 43, 509-520.

McGrath, S., Neve, H., Seegers, J. F., Eijlander, R., Vegge, C. S., Brøndsted, L., et al. (2006). Anatomy of a lactococcal phage tail. Journal of Bacteriology, 188, 3972-3982.

Moineau, S., Pandian, S., \& Klaenhammer, T. R. (1994). Evolution of a lytic bacteriophage via DNA acquisition from the Lactococcus lactis chromosome. Applied and Environmental Microbiology, 60, 1832-1841.

Muhammed, M. K., Olsen, M. L., Kot, W., Neve, H., Castro-Mejía, J. L., Janzen, et al. (2018). Investigation of the bacteriophage community in induced lysates of undefined mesophilic mixed-strain dl-cultures using classical and metagenomic approaches. International Journal of Food Microbiology, 272, $61-72$.

Pedersen, M., Ostergaard, S., Bresciani, J., \& Vogensen, F. K. (2000). Mutational analysis of two structural genes of the temperate lactococcal bacteriophage TP901-1 involved in tail length determination and baseplate assembly. Virology, 276, 315-328.

Penadés, J. R., Chen, J., Quiles-Puchalt, N., Carpena, N., \& Novick, R. P. (2015). Bacteriophage-mediated spread of bacterial virulence genes. Current Opinion in Biotechnology, 23, 171-178.

Smid, E. J., Erkus, O., Spus, M., Wolkers-Rooijackers, J. C., Alexeeva, S., \& Kleerebezem, M. (2014). Functional implications of the microbial community structure of undefined mesophilic starter cultures. Microbial Cell Factories, 13, Article S2.

Spus, M., Li, M., Alexeeva, S., Wolkers-Rooijackers, J., Zwietering, M., Abee, T., et al. (2015). Strain diversity and phage resistance in complex dairy starter cultures. Journal of Dairy Science, 98, 5173-5182.

Stern, A., \& Sorek, R. (2011). The phage-host arms race: Shaping the evolution of microbes. BioEssays, 33(1), 43-51.

Stockdale, S. R., Collins, B., Spinelli, S., Douillard, F. P., Mahony, J., Cambillau, C., et al. (2015). Structure and assembly of TP901-1 virion unveiled by mutagenesis. PloS One, 10, Article e0131676.

Tamura, K., \& Nei, M. (1993). Estimation of the number of nucleotide substitutions in the control region of mitochondrial DNA in humans and chimpanzees. Molecular Biology and Evolution, 10, 512-526.

Tamura, K., Stecher, G., Peterson, D., Filipski, A., \& Kumar, S. (2013). MEGA6: Molecular evolutionary genetics analysis version 6.0. Molecular Biology and Evolution, 30, 2725-2729.

UniProt Consortium. (2019). UniProt: A worldwide hub of protein knowledge. Nucleic Acids Research, 47, D506-D515.

van Sinderen, D., Karsens, H., Kok, J., Terpstra, P., Ruiters, M., Venema, G., et al. (1996). Sequence analysis and molecular characterization of the temperate lactococcal bacteriophage r1t. Molecular Microbiology, 19, 1343-1355.

Veesler, D., \& Cambillau, C. (2011). A common evolutionary origin for tailedbacteriophage functional modules and bacterial machineries. Microbiology and Molecular Biology Reviews, 75, 423-433.

Veesler, D., Spinelli, S., Mahony, J., Lichiere, J., Blangy, S., Bricogne, G., et al. (2012). Structure of the phage TP901-1 1.8 MDa baseplate suggests an alternative host adhesion mechanism. Proceedings of the National Academy of Sciences of the United States of America, 109, 8954-8958.

Vegge, C. S., Brøndsted, L., Neve, H., Mc Grath, S., van Sinderen, D., \& Vogensen, F. K. (2005). Structural characterization and assembly of the distal tail structure of the temperate lactococcal bacteriophage TP901-1. Journal of Bacteriology, 187, 4187-4197.

Ventura, M., Zomer, A., Canchaya, C., O'Connell-Motherway, M., Kuipers, O., Turroni, F., et al. (2007). Comparative analyses of prophage-like elements present in two Lactococcus lactis strains. Applied and Environmental Microbiology, $73,7771-7780$ 